\title{
Completion of FCVRP using Hybrid Particle Swarm Optimization Algorithm
}

\author{
Muhammad Ali a*, Baiq Nurul Izzah Farida ${ }^{b}$ \\ a School of Management, University of Western Sydney, Penrith South DC, Australia \\ Address: Second Ave, Kingswood NSW 2747, Australia \\ b Department of Industrial Management, National Formosa University, Taiwan \\ Address: No. 64, Wenhua Road, Huwei Township, Yunlin County, Taiwan \\ * Corresponding author: ali_muhammad32@gmail.com
}

\section{ARTICLE INFO}

Article history

Received September 12, 2020

Revised February 26, 2021

Accepted February 27, 2021

Available Online February 28, 2021

\section{Keywords}

Fuel consumption

Particle swarm

Vehicle

Routing

FCVRP

\begin{abstract}
The issue of green logistics has received full attention from the government and business people. It is closely related to the increase in Green House Gas (GHG) by transportation activities in the logistics sector. Controlling fuel consumption in transportation activities is fundamental in dealing with GHG. Therefore, the Fuel Consumption Vehicle Routing Problem (FCVRP) is proposed as a solution model in optimizing fuel consumption in the logistics sector. This study aims to develop a Hybrid Particle Swarm Optimization (HPSO) algorithm to solve the FCVRP problem. The proposed algorithm is the development of the PSO algorithm with local procedures search. Several experiments were carried out to determine the HPSO parameter's effect on minimizing fuel consumption in the FCVRP. The experiment results show that increasing the population and iteration parameters can produce minimum fuel consumption. Furthermore, the smaller the total fuel consumption produced when the kilometers per liter (KPL) high.
\end{abstract}

This is an open-access article under the CC-BY-SA license.

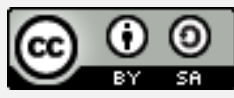

\section{Introduction}

Recently, the government and business players have increased their awareness of green logistics in the industrial sector [1]. The background of concern is based on the fact that logistics activities cause significant negative impacts on the environment [2]. Transportation is the most critical element in logistics and infrastructure fundamental to economic growth [3]. However, transportation also contributes to a large part of overall pollution [4]. In the last few decades, scientists have revealed that transportation activity causes an increase in Green House Gas (GHG) in the atmosphere [5]. Thus, the industrial sector's logistic policies need to consider environmental and ecological effects and focus on economic aspects.

According to Poonthalir and Nadarajan [6], fuel consumption in transportation activities is an essential parameter in controlling GHG. One of the main parameters affecting fuel consumption is the weight of load [7]. According to the US Department of Energy, Wang and Kuo [8] stated that fuel consumption increased by $2 \%$ for each additional 100-pound capacity. So that in this situation, logistics activities require a 
settlement model to minimize fuel consumption. One solution to this problem is to use the Green Vehicle Routing Problem (GVRP) [9]. GVRP can effectively increase fuel and pollutant efficiency by planning the proper routing of vehicles [10]. The GVRP variant demonstrating the importance of minimizing fuel consumption is called the Fuel Consumption Vehicle Routing Problem (FCVRP) [11].

Several studies have considered environmental aspects in most of the GVRP research. In this area, FCVRP is a new extension that reduces fuel consumption to reduce environmental impact. This study was first presented by Suzuki [12] by applying the Search Tabu (TS) to search for a solution. Xiao and Konak [13] completed the FCVRP model with the Simulated Annealing Algorithm. Several other solution approaches were used to solve the FCVRP problem, including intelligence heuristic partitioning by Gaur, et al. [7]. A gravitational search algorithm by MirHassani and Mohammadyari [14] to solve FCVRP. Several algorithms were proposed in other studies, such as the genetic algorithm proposed by Psychas, et al. [15], a firefly algorithm developed by Zhang, et al. [16]. In their study, Rao, et al. [17] used the Local Search hybrid to solve the FCVRP model. Meanwhile, Niu, et al. [18] utilized a novel hybrid Tabu Search algorithm.

Another study aimed to minimize the fuel consumption of a vehicle was presented by Kuo [19]. Kuo used the Simulated Annealing Algorithm to solve the time-dependent VRP. Xiao, et al. [20] also implemented a Simulated Annealing Algorithm. Other algorithms that have been used for this purpose were the genetic algorithm [21] and the ant colony algorithm [22]. Peng and Wang [23] also participated in the development of the FCVRP. Psychas, et al. [24] proposed a differential evolution algorithm, Eydi and Alavi [25] developed a mixed-integer linear programming model as a solution approach. The particle swarm optimization algorithm was used by Poonthalir and Nadarajan [6] to minimize the fuel consumption of the GVRP model. In the same model, Utama, et al. [3] utilized the hybrid butterfly optimization algorithm's development. Meanwhile, Dewi and Utama [10] proposed the development of a hybrid whale optimization algorithm. PSO was also used by Norouzi, et al. [26] to minimize fuel consumption with a time dependency. Research by Normasari, et al. [27] and Wang, et al. [28] used a Simulated Annealing Algorithm, slightly different from Andelmin and Bartolini [29], who chose to use the local search procedure. Macrina, et al. [30] proposed an extensive neighborhood hybrid as a way of finding solutions. In contrast, Wang and Lu [31] proposed a picking algorithm. Another study by Zhang, et al. [32] exercised the Tabu Search development.

Although research on GVRP continues to increase, studies related to FCVRP are still very limited. Research on this subject is mostly limited to the aspects of vehicle speed and travel distance. However, there is very little exploration of FCVRP research that focuses on the weight of the load. Looking at the gab, this study focuses on minimizing fuel consumption in the FCVRP study by considering the load aspect's weight. In addition, to our knowledge, there has been no previous research investigating studies related to FCVRP with the hybrid particle swarm optimization (HPSO) approach. Therefore, this research aims to minimize fuel consumption with HPSO as a solution approach. The contribution of this research is to propose a new hybrid particle swarm optimization procedure to solve the FCVRP problem. In addition, this study provides a careful investigation of the effect of kilometers per liter (KPL) on FCVRP.

This research's structure is presented as follows; Section (2) Method presents assumptions, notation and description of the problem, hybrid particle swarm optimization algorithm, and data and experiments. Section (3) Results and Discussion describes several topics such as hybrid particle swarm optimization solutions and changes in KPL on total fuel consumption. The last section is the conclusion of the entire research series and suggestions for further research. 


\section{Methods}

\subsection{Assumptions, Notations, and Problem Descriptions}

The problem assumptions in the FCVRP problem are as follows; (1) Each customer is only served by one vehicle, (2) Each vehicle departs and returns to the depot (3) Fuel consumption is affected by the weight of the load, KPL, and distance (4) Vehicles for distribution activities are homogeneous (4) Demand for every customer is a regular.

To describe the FCVRP problem, this study uses the notations described as follows:

$\mathrm{KPL}$ : distance traveled per unit of fuel

$p \quad$ : fuel consumption increases with each additional load

$k \quad$ : increase in load capacity

$L_{i j} \quad:$ load

$K \quad$ : collection of vehicles at the depot, with $K=\{1,2, \ldots, k$

$R^{k} \quad:$ set of routes traveled by the vehicle $k$, with $R=\left\{R^{1}, R^{2}, \ldots, R^{k}\right\}$

$V \quad$ : set node

$d_{i j} \quad:$ distance from $i$ to $j$ nodes

$r \quad$ : route index

$C_{r}^{k} \quad$ : operational costs for vehicle $k$ traveling the route $r$

$A_{r i}^{k} \quad$ : constant, value 1 if vehicle $k$ with route $r$ to customer $i$ and value 0 for other conditions

$T_{k}^{r} \quad$ : time taken for the vehicle to travel the route $r$

$T_{\max }:$ maximum allowable travel time

FC : Total Fuel Consumption

$R_{r}^{s} \quad: s$ th node in $r$ th route, for example $R_{2}^{4}=4$ can be interpreted as the 4 th node on the 2 nd route is 4 (0-5-2-3-4-0)

In this study, the problem description was described in a mathematical model. The objective function of this FCVRP problem was to minimize fuel consumption. The mathematical model that described the problem description is described as follows:

Objective function:

Minimize $F C=\sum_{v \in V} \sum_{r \in R^{v}} \frac{d_{i j}}{K P L_{i j}}\left(1+p\left(L_{i j} / k\right)\right)_{r}^{k} x_{r}^{v}$

Constraint:

$\sum_{k \in K} \sum_{r \in R^{k}} C_{r}^{k} x_{r}^{k} \geq 1$

$\forall i \in N$

$\sum_{k=1}^{K} y_{i k}=1$

$\forall i \in V\{0\}$

$\sum_{k=1}^{K} y_{0 k}=K$

$\sum_{i \in V} d_{i} y_{i k} \leq C_{k}$

$\forall k=1,2, \ldots K$

$\sum x_{r}^{k} \geq 1$

$\forall k \in K$

$\sum_{r \in R^{k}} T_{r}^{k} X_{r}^{k} \leq T_{\text {Max }}$

$\forall \mathrm{k} \in \mathrm{K}$

$x_{r}^{k} \in\{0,1\}$

$\forall k \in K, \forall r \in R^{k}$

With the decision variables:

$x_{r}^{k}= \begin{cases}1, & \text { If vehicle } k \text { using } r \text { route } \\ 0, & \text { Other }\end{cases}$ 
Equation (1) was the objective function to minimize fuel consumption. The constraint in Equation (2) was ensuring that all customers can be visited. Equation (3) guaranteed that each customer was served once by a vehicle. The limitation in equation (4) was to guarantee that $\mathrm{K}$ vehicles could carry out distribution activities. The constraint in equation (5) ensured that customer demand did not exceed the vehicle capacity on each route. Equation (6) was ensuring that every vehicle passed at least one lane/route. The constraint in equation (7) was to ensure that the vehicle travel time did not exceed the allowable travel time. Equation (8) was a constraint that guaranteed the decision variable $x_{r}^{k}$ was a binary integer.

\subsection{FCVRP Completion with Hybrid Particle Swarm Optimization (HPSO) Algorithm}

This study suggested the use of the HPSO algorithm to minimize fuel consumption. The HPSO algorithm is a model development of the Particle Swarm Optimization (PSO) algorithm. The proposed algorithm combines PSO with a local search strategy. The PSO algorithm was initially proposed by Trelea [33] in 2003. Each particle has characteristics that distinguish PSO from other algorithms, namely position and velocity. NP-hard problems such as FCVRP require high computation time in line with the complexity of the study. The hybrid process in an algorithm can increase the effectiveness of finding a solution. Therefore, Hybrid PSO (HPSO) was proposed in this study to minimize fuel consumption in FCVRP problems.

HPSO has stages in its journey, such as (1) Converting particle positions into travel routes using the Short Rank Value (SRV) method (2) Updating inertia weight (3) Updating cognitive acceleration (4) Updating social acceleration (5) Updating velocity (6) Updating particle position (7) Conducting local search. The local search procedure used to develop the algorithm was the swap and flip procedure. The following is an explanation regarding the stages of HPSO in completing the FCVRP.

\subsubsection{Convert particle position into travel route}

The search for a solution began with initializing the position through a random number with an upper limit (ub) and a lower limit (lb). The upper limit (ub) and the lower limit (lb) were used to determine each particle's position. In this process, there should be no repetition of values in each position dimension. This illustration is presented in Fig. 1. The position value (continuous) was then converted into a trip sequence (discrete) using the SRV method. The way these method works was to sort the position values from the smallest to the largest value. Fig. 2 shows a representation of the SRV process.

$$
P=\left\{\begin{array}{lll}
0,56 & 0,91 & 0,85 \\
0,77 & 0,39 & 0,75 \\
0,42 & 0,12 & 0,55
\end{array}\right\} \quad P=\left\{\begin{array}{lll}
0,56 & 0,91 & 0,85 \\
0,77 & 0,91 & 0,75 \\
0,42 & 0,12 & 0,55
\end{array}\right\}
$$

Fig. 1. Initialization of the position value (a) accepted population (b) rejected population 


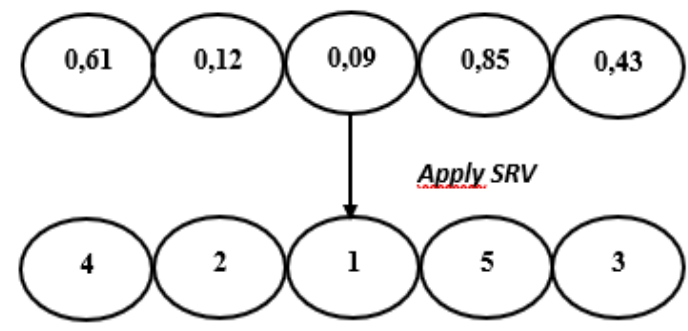

(A)

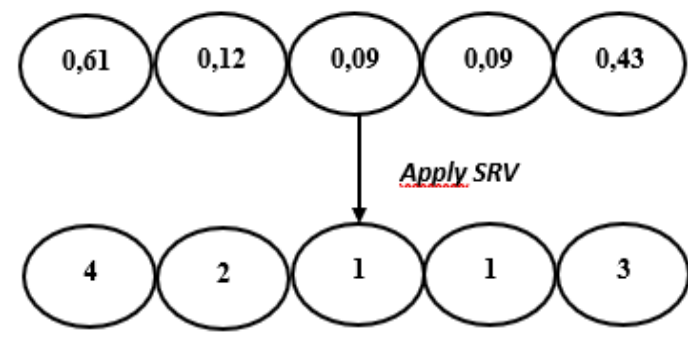

(B)

Fig. 2. SRV application (a) correct travel sequence (b) wrong travel sequence

The procedure for calculating fuel consumption can be seen in Fig. 3. The formula for calculating the fuel consumption was carried out by reversing the sequence of the subroutes formed. Fig. 3 describes the sum of all customer requests distributed from the customer $R_{n}^{(e)}$ and followed by the next customer in reverse until customer $R_{n}^{(s)}$ and the last one visited was the distribution center.

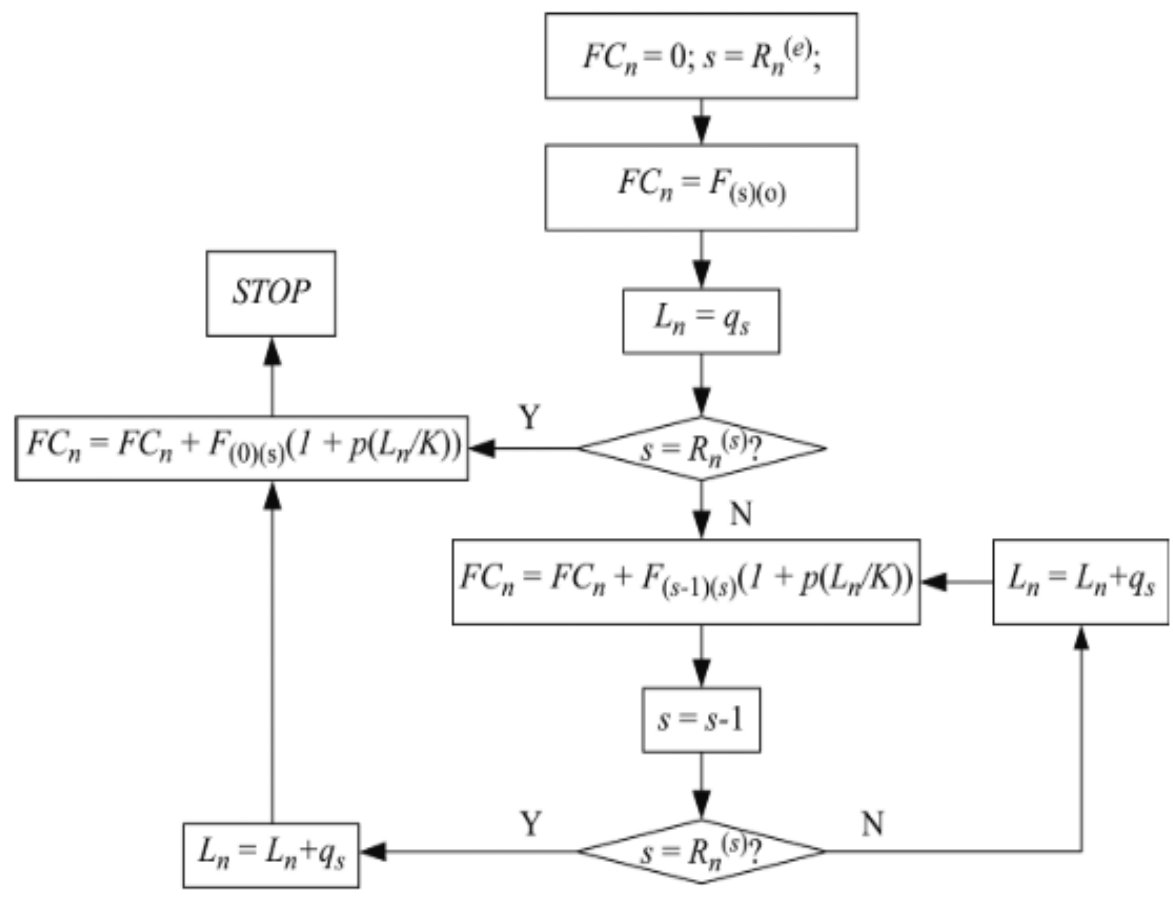

Fig. 3. Fuel Calculation Procedure

\subsubsection{Update inertia weight, cognitive acceleration, dan social acceleration}

One of the factors that affect an algorithm's performance in solving an optimization problem is determining the right combination of parameters. The particle size used refers to Eberhart and Yuhui [34], namely 30-50 particles.

The value of inertia weight $(\omega)$ and the coefficient acceleration $\left(c_{1}\right.$ and $\left.c_{2}\right)$ referred to Ratnaweera, et al. [35]. The value of $\omega_{\max }=0,9, \omega_{\min }=0,4, c_{1 \max }=0.5, c_{1 \min }=2.5$, $c_{2 \min }=0.5, c_{2 \max }=2.5$. Furthermore, the initial velocity of each particle was assumed to be $v_{0}=0$. The maximum particle velocity was $v_{\max }=20$. Ratnaweera, et al. [35] have suggested this method carried out with a factor inertia weight $(\omega)$ (equation 9) and coefficient acceleration $\left(c_{1}\right.$ and $c_{2}$ ) (equation 10 dan 11) which varies with time. 
$\omega_{\text {curr_iter }}=\left(\omega_{\max }-\omega_{\text {min }}\right) * \frac{\max \_ \text {iter-curr_iter }}{\max \_ \text {iter }}+\omega_{\text {min }}$

$c_{1}=\left(c_{1 \text { max }}-c_{1 \text { min }}\right) * \frac{\text { curr_iter }}{\text { max _iter }}+c_{1 \text { min }}$

$c_{2}=\left(c_{2 \max }-c_{2 \min }\right) * \frac{\text { curr_iter }}{\text { max _iter }}+c_{2 \min }$

\subsubsection{Update velocity, particle position}

Each particle was assumed to have two characteristics; position and velocity. Each particle moves in a certain space and remembers the best position ever traveled or found against a food source or objective function value. Each particle conveys information or its good position to the other particles and adjusts each position and velocity.

The following is a mathematical formulation that describes the position and velocity of particles in a search space.

$$
\begin{aligned}
& v_{i(t+1)}=V_{i(t)}+c_{1} r_{1}\left(p_{\text {best }(i)}-x_{i(t)}\right)+c_{2} r_{2}\left(g_{\text {best }(i)}-x_{1(t)}\right) \\
& x_{i(t+1)}=v_{i(t+1)}+x_{i(t)}
\end{aligned}
$$

$p_{\text {best }(i)}=p_{\text {best }(i 1)}, p_{\text {best }(i 2)}, p_{\text {best }(i 3)}, \ldots, p_{\text {best }(i N)}$ represents the local best of the $\mathrm{i}(\mathrm{th})$ particle. Whereas $g_{\text {best }(i)}=g_{\text {best }(i 1)}, g_{\text {best }(i 2)}, g_{\text {best }(i 3)}, \ldots, g_{\text {best }(i N)}$ represents the global best of all particles. $c_{1}$ and $c_{2}$ are the acceleration coefficients which are positive, then $r_{1}$ and $r_{2}$ is a random number whose value is between 0 and 1 .

\subsubsection{Local search}

To improve the PSO algorithm's performance, this study proposed PSO with the local search procedure. Some of the procedures used to maximize the efficiency of the PSO were the swap and flip procedures. Fig. $4 \mathrm{~b}$ illustrates the stages of the swap procedure. The swap procedure begins by selecting two positions or nodes randomly and then swapping positions. On the other hand, the flip procedure has steps where two nodes are randomly selected. Then the selected nodes are reversed in order. The stages of the flip procedure are described in Figure 4a. The implementation of the hybrid in PSO with swap and flip procedures was carried out in each iteration $t$ as many as the number of nodes. Algorithm 1 and Algorithm 2 show the pseudocode for the PSO and HPSO algorithms.

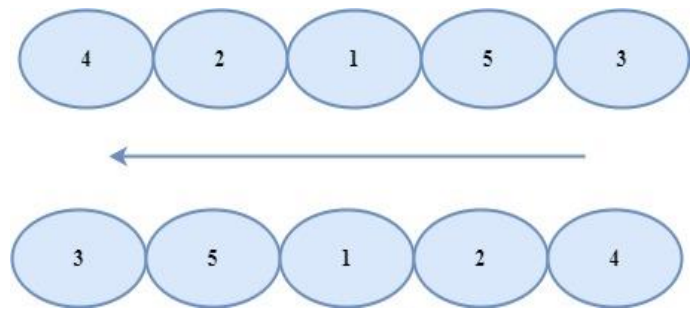

(a)

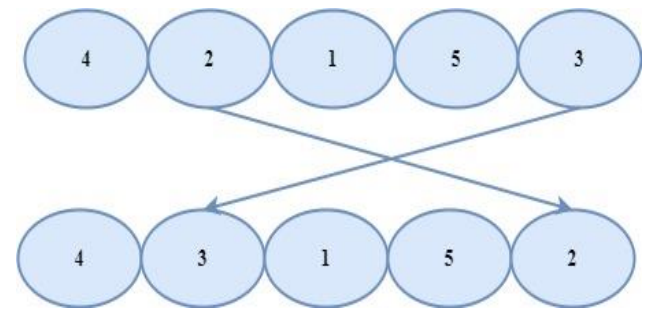

(b)

Fig. 4. (a) flip ilustration (b) swap ilustration

To get optimal performance, Ratnaweera, et al. [35] reduce the cognitive component's value and increase the social component by changing the acceleration coefficient $\mathrm{c} 1$ and $\mathrm{c} 2$ along with the iteration. The larger the value of $\mathrm{c} 1$ and the smaller 
the value of $\mathrm{c} 2$, in the beginning, means the particles are allowed to move around the search space and move towards the best population. On the other hand, the small cognitive component's value and the large social component allow the particles to converge towards global optima at the end of the optimization.

Algorithm 1: Pseudocode Particle Swarm Optimization (PSO)

\section{Algoritma PSO}

\section{Initialization}

Select the variant of the PSO algorithm

Select parameters of the PSO $\left(\mathrm{w}_{1}, \mathrm{c}_{1 \max }, \mathrm{c}_{1 \min }, \mathrm{c}_{2 \max }, \mathrm{c}_{1 \min }, \mathrm{v}_{\max }, \mathrm{n}\right.$, max iteration (t))

Initialize the routes with a random way [position=rand.* $(\mathrm{ub}-\mathrm{lb})+\mathrm{lb}$ ]

Convert particle' position in continuous form

Initialize the position and velocity of each particle

Convert particle' position into routing division (section 2.2.2)

Calculate the initial fitness function of each particle in section 2.4.1 and eq (11)

while $\left(t<t_{\max }\right)$

do for each particle

for each task

do calculate the particle's fitness value according to equation (11)

do if fitness value $<\mathrm{P}_{\text {best }}$

end if

$$
\text { then } \mathrm{P}_{\text {best }}=\text { fitness }
$$

do if fitness value $<\mathrm{G}_{\text {best }}$

end if

$$
\text { then } G_{\text {best }}=\text { fitness }
$$

do updating inertia weight according to equation (9)

do updating cognitive acceleration $c_{1}$ according to equation (10)

do update the social acceleration $c_{2}$ according to equation (11)

do update the particle's velocity according to the equation (12)

do update the particle's position according to the equation (13)

end for

end for

end while

\subsection{Data Collection and Experiment}

\subsubsection{Data Collection}

In this study, several numbers nodes were used as a numerical experiment. The coordinates of customer position, number of customer requests, and capacity were based on the problems of Gaskell [36] and Dantzig and Ramser [37]. Nodes 21 and 22 were taken from Gaskell's data set [36], and node 12 was derived from the Dantzig and Ramser problem [37]. The distance between customers and the distance from the depot to the customer $\left(d_{\left(R_{r}^{n}\right)\left(R_{r}^{n+1}\right)}\right)$ were calculated by the euclidian distance formula in equation (14).

$$
d_{\left(R_{r}^{n}\right)\left(R_{r}^{n+1}\right)}=\sqrt{\left(X_{n}-X_{n+1}\right)^{2}+\left(Y_{n}-Y_{n+1}\right)^{2}}
$$


Algorithm 2: Pseudocode Hybrid Particle Swarm Optimization (HPSO)

\section{Algoritma HPSO}

\section{Initialization}

Select the variant of the PSO algorithm

Select parameters of the PSO $\left(\mathrm{w}_{1}, \mathrm{c}_{1 \max }, \mathrm{c}_{1 \min }, \mathrm{c}_{2 \max }, \mathrm{c}_{1 \min }, \mathrm{v}_{\max }, \mathrm{n}\right.$, max iteration $\left.(\mathrm{t})\right)$

Initialize the routes with a random way [position=rand.* $(u b-l b)+l b]$

Convert particle' position in continuous form

Initialize the position and velocity of each particle

Convert particle' position into routing division (section 2.2.2)

Calculate the initial fitness function of each particle in section 2.4.1 and eq (11)

while $\left(t<t_{\max }\right)$

do for each particle

for each task

do calculate the particle's fitness value according to equation (11)

do if fitness value $<\mathrm{P}_{\text {best }}$

end if

$$
\text { then } P_{\text {best }}=\text { fitness }
$$

do if fitness value $<G_{\text {best }}$

end if

then $G_{\text {best }}=$ fitness

do updating inertia weight according to equation (9)

do updating cognitive acceleration $c_{1}$ according to equation (10)

do update the social acceleration $c_{2}$ according to equation (11)

do update the particle's velocity according to the equation (12)

do update the particle's position according to the equation (13)

end for

end for

Apply local search

For $\mathrm{i}=1$; node

Perform swap on the $X^{*}$. Ensure No. repeated swap in the $X^{*}$

If $\mathrm{Xt}<\mathrm{X}^{*}$

$\mathrm{X}^{*}<\mathrm{Xt}$

end if

end for

For $\mathrm{j}=1$; node

Perform flips on the $\mathrm{X}^{*}$. Ensure No. repeated flip in the $\mathrm{X}^{*}$

If $\mathrm{Xt}<\mathrm{X}^{*}$

$\mathrm{X}^{*}<\mathrm{Xt}$

end if

end for

end while

Here, $r$-th was the route consisting of node $s$ to node $s+1$. KPL calculation or distance per unit of fuel refers to Kuo's research [19]. The increase in fuel consumption $(\rho)$ at each additional load per 100 pounds or 45.35 kilograms was $2 \%$. This study was conducted in 9 variations; three variations in the number of nodes and three variations in 
the value of the vehicle KPL. Table 1 shows the distance matrix from node 12 , while Table 2 and Table 3 show the position coordinates of nodes 21 and 22 .

Table 1 . The distance matrix from 12 node

\begin{tabular}{ccccccccccccccc}
\hline Node & 0 & 1 & 2 & 3 & 4 & 5 & 6 & 7 & 8 & 9 & 10 & 11 & 12 & demand \\
\hline 0 & 0 & 9 & 14 & 21 & 23 & 22 & 25 & 32 & 36 & 38 & 42 & 50 & 52 & 1200 \\
\hline 1 & 9 & 0 & 5 & 12 & 22 & 21 & 24 & 31 & 35 & 37 & 41 & 49 & 51 & 1700 \\
\hline 2 & 14 & 5 & 0 & 7 & 17 & 16 & 23 & 26 & 30 & 36 & 36 & 44 & 51 & 1500 \\
\hline 3 & 21 & 12 & 7 & 0 & 10 & 21 & 30 & 27 & 37 & 43 & 31 & 37 & 39 & 1400 \\
\hline 4 & 23 & 22 & 17 & 10 & 0 & 19 & 28 & 25 & 35 & 41 & 29 & 31 & 29 & 1700 \\
\hline 5 & 22 & 21 & 16 & 21 & 19 & 0 & 9 & 10 & 16 & 22 & 20 & 28 & 30 & 1400 \\
\hline 6 & 25 & 24 & 23 & 30 & 28 & 9 & 0 & 7 & 11 & 13 & 17 & 25 & 27 & 1200 \\
\hline 7 & 32 & 31 & 26 & 27 & 25 & 10 & 7 & 0 & 10 & 16 & 10 & 18 & 20 & 1200 \\
\hline 8 & 36 & 35 & 30 & 37 & 35 & 16 & 11 & 10 & 0 & 6 & 6 & 14 & 16 & 1900 \\
\hline 9 & 38 & 37 & 36 & 43 & 41 & 22 & 13 & 16 & 6 & 0 & 12 & 12 & 20 & 1800 \\
\hline 10 & 42 & 41 & 36 & 31 & 29 & 20 & 17 & 10 & 6 & 12 & 0 & 8 & 10 & 1600 \\
\hline 11 & 50 & 49 & 44 & 37 & 31 & 28 & 25 & 18 & 14 & 12 & 8 & 0 & 10 & 1700 \\
\hline 12 & 52 & 51 & 51 & 39 & 29 & 30 & 27 & 20 & 16 & 20 & 10 & 10 & 0 & 1100 \\
\hline Capacity & 6000 & & & & & & & & & & & & & \\
\hline
\end{tabular}

Table 2. The coordinates of the position of 21 node problem

\begin{tabular}{|c|c|c|c|}
\hline No & $\mathrm{x}$ & $\mathrm{y}$ & Demand \\
\hline 0 & 145 & 215 & 0 \\
\hline 1 & 151 & 264 & 1100 \\
\hline 2 & 159 & 261 & 700 \\
\hline 3 & 130 & 254 & 800 \\
\hline 4 & 128 & 252 & 1400 \\
\hline 5 & 163 & 247 & 2100 \\
\hline 6 & 146 & 246 & 400 \\
\hline 7 & 161 & 242 & 800 \\
\hline 8 & 142 & 239 & 100 \\
\hline 9 & 163 & 236 & 500 \\
\hline 10 & 148 & 232 & 600 \\
\hline 11 & 128 & 231 & 1200 \\
\hline 12 & 156 & 217 & 1300 \\
\hline 13 & 129 & 214 & 1300 \\
\hline 14 & 146 & 208 & 300 \\
\hline 15 & 164 & 208 & 900 \\
\hline 16 & 141 & 206 & 2100 \\
\hline 17 & 147 & 193 & 1000 \\
\hline 18 & 164 & 193 & 900 \\
\hline 19 & 129 & 189 & 2500 \\
\hline 20 & 155 & 185 & 1800 \\
\hline 21 & 139 & 182 & 700 \\
\hline Capaity & 6000 & & \\
\hline
\end{tabular}


Table 3. Coordinates of the position of 22 node problem

\begin{tabular}{|c|c|c|c|}
\hline node & $\mathrm{x}$ & $\mathrm{y}$ & demand \\
\hline 0 & 266 & 235 & 0 \\
\hline 1 & 295 & 272 & 125 \\
\hline 2 & 301 & 258 & 84 \\
\hline 3 & 309 & 260 & 60 \\
\hline 4 & 217 & 274 & 500 \\
\hline 5 & 218 & 278 & 300 \\
\hline 6 & 282 & 267 & 175 \\
\hline 7 & 242 & 249 & 350 \\
\hline 8 & 230 & 262 & 150 \\
\hline 9 & 249 & 268 & 1100 \\
\hline 10 & 256 & 267 & 4100 \\
\hline 11 & 265 & 257 & 225 \\
\hline 12 & 267 & 242 & 300 \\
\hline 13 & 259 & 265 & 250 \\
\hline 14 & 315 & 233 & 500 \\
\hline 15 & 329 & 252 & 150 \\
\hline 16 & 318 & 252 & 100 \\
\hline 17 & 329 & 224 & 250 \\
\hline 18 & 267 & 213 & 120 \\
\hline 19 & 275 & 192 & 600 \\
\hline 20 & 303 & 201 & 500 \\
\hline 21 & 208 & 217 & 175 \\
\hline 22 & 326 & 181 & 75 \\
\hline Capacity & 4500 & & \\
\hline
\end{tabular}

\subsubsection{Setup the experiment}

In this study, an experiment was conducted to determine the vehicle's fuel consumption's HPSO parameters' performance. The calculation experiment was carried out with variations in the number of populations and iterations. Variations in population parameters used were 30,40 , and 50 populations. Meanwhile, the iteration parameters used in the experiment varied with a range of 10-300 iterations. The calculation experiment was carried out in as many as 54 experiments. Each calculation result was performed a recapitulation of the fuel consumption results. The recapitulation of the overall experimental results was analyzed to determine population variations and iterations on numerical experiments with variations in the number of nodes.

In this study, an analysis of the Kilometers Per Liter (KPL) effect on fuel consumption was also conducted. KPL was tested with a value of 9.35, 12.8, and 16.25. Each experimental result was recorded and analyzed for the effect of the changes on the fuel consumption result. All calculation experiments were carried out using Matlab 2014a software on Windows 10 AMD A12 with x64-64 8GB RAM processor. 


\section{Results and Discussion}

\subsection{Solution Using HPSO}

In this section, the results of the experimental calculation of the FCVRP study using the Hybrid Particle Swarm Optimization (HPSO) approach were explained. The fuel consumption calculation was carried out in 3 different cases. Table 4, Table 5, and Table 6 portray the experiment calculation results with variations in the number of nodes, population, and iteration.

Table 4. Fuel consumption (liter) from 12 node

\begin{tabular}{ccccccc}
\hline \multirow{2}{*}{ Population } & \multicolumn{6}{c}{ Iteration } \\
\cline { 2 - 7 } & 10 & 30 & 50 & 100 & 200 & 300 \\
\hline 30 & 35.08 & 34.7578 & 32.6578 & 34.62 & 34.408 & 31.758 \\
\hline 40 & 34.433 & 33.1703 & 33.3578 & 33.986 & 33.592 & 34.014 \\
\hline 50 & 36.883 & 34.9766 & 33.2734 & 32.658 & 31.758 & 31.758 \\
\hline
\end{tabular}

Table 5. Fuel consumption (liter) from 21 node

\begin{tabular}{ccccccc}
\hline \multirow{2}{*}{ Population } & \multicolumn{5}{c}{ Iteration } \\
\cline { 2 - 7 } & 10 & 30 & 50 & 100 & 200 & 300 \\
\hline 30 & 67.988 & 67.11 & 65.617 & 57.3616 & 54.463 & 51.05 \\
\hline 40 & 68.259 & 59.294 & 56.306 & 54.2321 & 54.271 & 50.723 \\
\hline 50 & 56.306 & 54.2321 & 54.271 & 50.723 & 50.894 & 48.2741 \\
\hline
\end{tabular}

Table 6. Fuel consumption (liter) from 22 node

\begin{tabular}{ccccccc}
\multirow{2}{*}{ Population } & \multicolumn{6}{c}{ Iteration } \\
\cline { 2 - 7 } & 10 & 30 & 50 & 100 & 200 & 300 \\
\hline 30 & 99.164 & 99.164 & 99.164 & 99.164 & 99.164 & 73.248 \\
\hline 40 & 99.164 & 99.164 & 99.164 & 99.164 & 99.164 & 99.164 \\
\hline 50 & 115.7 & 84.664 & 74.560 & 99.164 & 99.164 & 62.329 \\
\hline
\end{tabular}

Table 4, Table 5, and Table 6 indicate that the optimal fuel consumption in the case of node 12 was obtained in an experiment with a population of 50 iterations of 300 . Like node 12 , the optimal fuel consumption in nodes 21 and 22 was obtained during the experiment on population 50 and iterations 300. Based on Table 6 , it is concluded that the optimal fuel consumption tends to be obtained in experiments with large population parameters and iterations. The greater the population parameter and the iteration, the greater the probability of getting optimal fuel consumption. Therefore, to minimize fuel consumption in this FCVRP case study, it is highly recommended to use various high parameters. Population parameters and high iterations appear to be more effective in generating minimum fuel consumption.

\subsection{Analysis of the Effect of Changes in KPL on Fuel Consumption}

The analysis was carried out on changes in the KPL variable on the fuel consumption. The procedure used was to experiment with calculating the KPL value, which varies from 9.35 to 16.25 liters. This process functioned to find the effect of changes in the value of the KPL variable on fuel consumption value. Fig. 5 shows that the greater 
the KPL variable's value, the smaller the total fuel consumption produced. On the other hand, the total fuel consumption was observed to get more significant when the KPL value gets smaller.

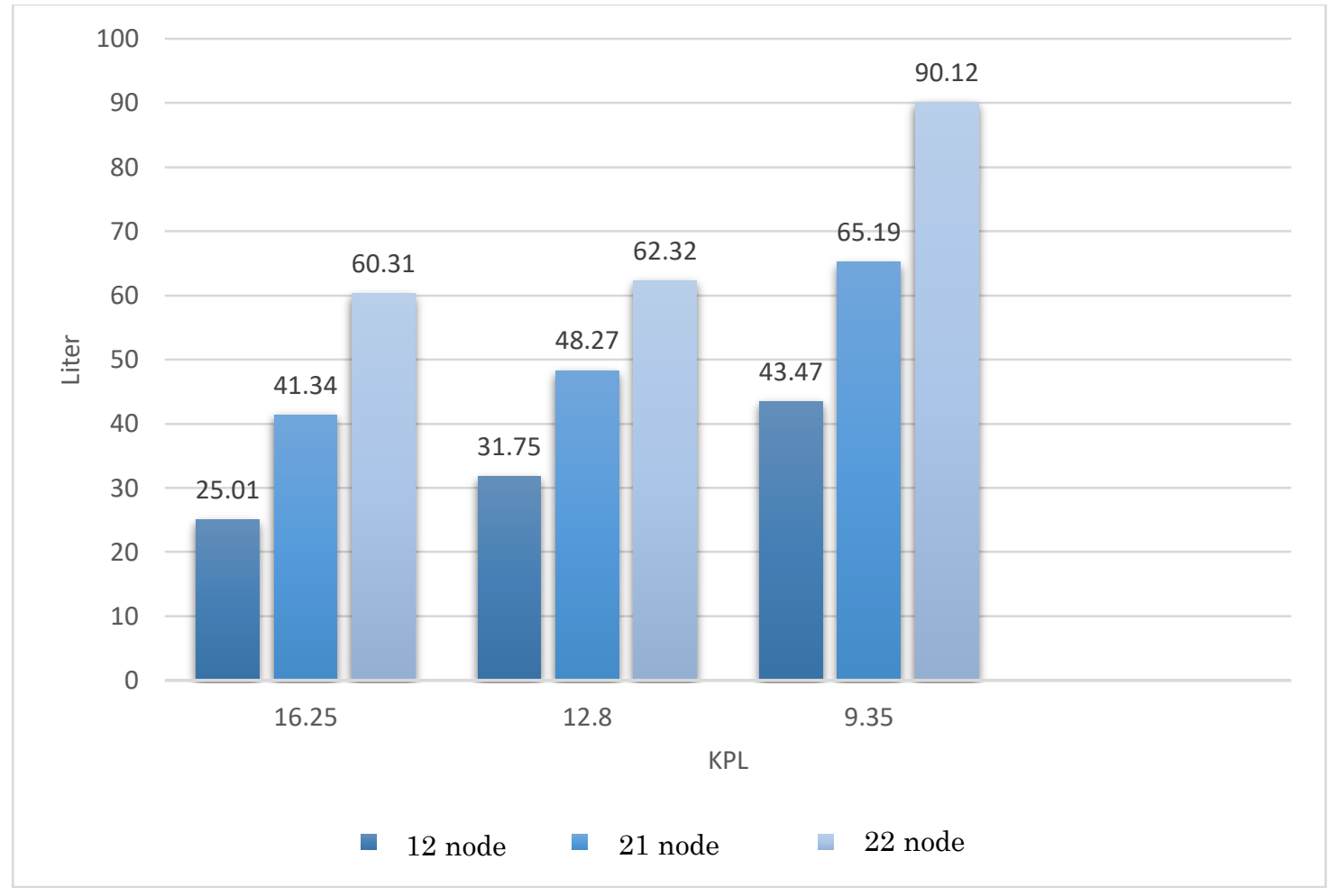

Fig. 5. Graph Analysis of the Effect of Changes in KPL variables on Fuel Consumption (FC)

\section{Conclusion}

This study discussed the problem of the Fuel Consumption Vehicle Routing Problem (FCVRP). The Hybrid Particle Swarm Optimization (HPSO) algorithm was developed to minimize fuel consumption in transportation activities. The experiment showed that to obtain a more optimal fuel consumption, the population and iteration parameters need to be increased. The effect of changes in KPL on energy consumption was also investigated. The results indicated that the greater the KPL variable's value, the smaller the total fuel consumption produced. Some of the limitations of this study included not considering the pick-up load at each node. The suggestion for further research is to investigate FCVRP problems by considering the pick-up and delivery load.

\section{Acknowledgments}

The researchers gratefully thank the faculty and university for their support in allowing this research to be carried out. Finally, the researchers thank the anonymous reviewers for their helpful suggestions.

\section{References}

[1] C. Lin, K. L. Choy, G. T. S. Ho, S. H. Chung, and H. Y. Lam, "Survey of Green Vehicle Routing Problem: Past and future trends," Expert Systems with 
$\begin{array}{lllll}\text { Applications, } & \text { vol. } & 41, \quad \text { pp. } & 1118-1138, & 2014 \text {. }\end{array}$ https://doi.org/10.1016/j.eswa.2013.07.107.

[2] Ç. Koç and I. Karaoglan, "The green vehicle routing problem: A heuristic based exact solution approach," Applied Soft Computing, vol. 39, pp. 154-164, 2016. https://doi.org/10.1016/j.asoc.2015.10.064.

[3] D. M. Utama, D. S. Widodo, M. F. Ibrahim, and S. K. Dewi, "A New Hybrid Butterfly Optimization Algorithm for Green Vehicle Routing Problem," Journal of Advanced Transportation, vol. 2020, p. 8834502, 2020. https://doi.org/10.1155/2020/8834502.

[4] K. Salimifard, H. Shahbandarzadeh, and R. Raeesi, "Green transportation and the role of operation research," in Int. Conf. Traffic Transp. Eng.(ICTTE 2012), 2012, pp. 74-79.

[5] A. Montoya, C. Guéret, J. E. Mendoza, and J. G. Villegas, "A multi-space sampling heuristic for the green vehicle routing problem," Transportation Research Part C: Emerging Technologies, vol. 70, pp. 113-128, 2016. https://doi.org/10.1016/j.trc.2015.09.009.

[6] G. Poonthalir and R. Nadarajan, "A Fuel Efficient Green Vehicle Routing Problem with varying speed constraint (F-GVRP)," Expert Systems with Applications, vol. 100, pp. 131-144, 2018. https://doi.org/10.1016/j.eswa.2018.01.052.

[7] D. R. Gaur, A. Mudgal, and R. R. Singh, "Routing vehicles to minimize fuel consumption," Operations Research Letters, vol. 41, pp. 576-580, 2013. https://doi.org/10.1016/j.orl.2013.07.007.

[8] C. C. Wang and Y. Kuo, "Optimizing the VRP by minimizing fuel consumption," Management of Environmental Quality: An International Journal, vol. 22, pp. 440450, 2011. https://doi.org/10.1108/14777831111136054.

[9] K. Karagul, Y. Sahin, E. Aydemir, and A. Oral, "A simulated annealing algorithm based solution method for a green vehicle routing problem with fuel consumption," in Lean and green supply chain management, ed: Springer, 2019, pp. 161-187. https://doi.org/10.1007/978-3-319-97511-5_6.

[10] S. K. Dewi and D. M. Utama, "A New Hybrid Whale Optimization Algorithm for Green Vehicle Routing Problem," Systems Science \& Control Engineering, vol. 9, pp. 61-72, 2021. https://doi.org/10.1080/21642583.2020.1863276.

[11] R. Moghdani, K. Salimifard, E. Demir, and A. Benyettou, "The green vehicle routing problem: A systematic literature review," Journal of Cleaner Production, vol. 279, p. 123691, 2021. https://doi.org/10.1016/j.jclepro.2020.123691.

[12] Y. Suzuki, "A new truck-routing approach for reducing fuel consumption and pollutants emission," Transportation Research Part D: Transport and Environment, vol. 16, pp. 73-77, 2011. https://doi.org/10.1016/j.trd.2010.08.003.

[13] Y. Xiao and A. Konak, "A simulating annealing algorithm to solve the green vehicle routing \& scheduling problem with hierarchical objectives and weighted tardiness," Applied Soft Computing, vol. 34, pp. 372-388, 2015. https://doi.org/10.1016/j.asoc.2015.04.054.

[14] S. A. MirHassani and S. Mohammadyari, "Reduction of carbon emissions in VRP by gravitational search algorithm," Management of Environmental Quality: An International Journal, vol. 25, pp. 766-782, 2014. https://doi.org/10.1108/MEQ-082013-0086.

[15] I.-D. Psychas, M. Marinaki, Y. Marinakis, and A. Migdalas, "Minimizing the fuel consumption of a multiobjective vehicle routing problem using the parallel multistart NSGA II algorithm," in International Conference on Network Analysis, 2014, pp. 69-88. https://doi.org/10.1007/978-3-319-29608-1_5. 
[16] Z. Zhang, L. Wei, and A. Lim, "An evolutionary local search for the capacitated vehicle routing problem minimizing fuel consumption under three-dimensional loading constraints," Transportation Research Part B: Methodological, vol. 82, pp. 20-35, 2015. https://doi.org/10.1016/j.trb.2015.10.001.

[17] W. Rao, F. Liu, and S. Wang, "An Efficient Two-Objective Hybrid Local Search Algorithm for Solving the Fuel Consumption Vehicle Routing Problem," Applied Computational Intelligence and Soft Computing, vol. 2016, p. 3713918, 2016. https://doi.org/10.1155/2016/3713918.

[18] Y. Niu, Z. Yang, P. Chen, and J. Xiao, "A Hybrid Tabu Search Algorithm for a RealWorld Open Vehicle Routing Problem Involving Fuel Consumption Constraints," Complexity, vol. 2018, p. 5754908, 2018. https://doi.org/10.1155/2018/5754908.

[19] Y. Kuo, "Using simulated annealing to minimize fuel consumption for the timedependent vehicle routing problem," Computers \& Industrial Engineering, vol. 59, pp. 157-165, 2010. https://doi.org/10.1016/j.cie.2010.03.012.

[20] Y. Xiao, Q. Zhao, I. Kaku, and Y. Xu, "Development of a fuel consumption optimization model for the capacitated vehicle routing problem," Computers \&

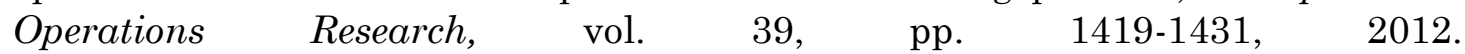
https://doi.org/10.1016/j.cor.2011.08.013.

[21] H. Xiong, "A Fuel Consumption Objective of VRP and the Genetic Algorithm," in 2010 International Conference on E-Product E-Service and E-Entertainment, 2010, pp. 1-4. https://doi.org/10.1109/ICEEE.2010.5661086.

[22] E. Yao, Z. Lang, Y. Yang, and Y. Zhang, "Vehicle routing problem solution considering minimising fuel consumption," IET Intelligent Transport Systems, vol. 9, pp. 523-529, 2015. https://doi.org/10.1049/iet-its.2015.0027.

[23] Y. Peng and X. Wang, "Research on a Vehicle Routing Schedule to Reduce Fuel Consumption," in 2009 International Conference on Measuring Technology and Mechatronics Automation, 2009, 8p. 825-827. https://doi.org/10.1109/ICMTMA.2009.11.

[24] I.-D. Psychas, M. Marinaki, Y. Marinakis, and A. Migdalas, "Non-dominated sorting differential evolution algorithm for the minimization of route based fuel consumption multiobjective vehicle routing problems," Energy Systems, vol. 8, pp. 785-814, 2017. https://doi.org/10.1007/s12667-016-0209-5.

[25] A. Eydi and H. Alavi, "Vehicle Routing problem in Reverse Logistics with Split Demands of customers and fuel consumption Optimization," Arabian Journal for Science and Engineering, vol. 44, pp. 2641-2651, 2019. https://doi.org/10.1007/s13369-018-3311-2.

[26] N. Norouzi, M. Sadegh-Amalnick, and R. Tavakkoli-Moghaddam, "Modified particle swarm optimization in a time-dependent vehicle routing problem: minimizing fuel consumption," Optimization Letters, vol. 11, pp. 121-134, 2017. https://doi.org/10.1007/s11590-015-0996-y.

[27] N. M. E. Normasari, V. F. Yu, and C. Bachtiyar, "A simulated annealing heuristic for the capacitated green vehicle routing problem," Mathematical Problems in Engineering, vol. 2019, pp. 1-18, 2019. https://doi.org/10.1155/2019/2358258.

[28] R. Wang, J. Zhou, X. Yi, and A. A. Pantelous, "Solving the green-fuzzy vehicle routing problem using a revised hybrid intelligent algorithm," Journal of Ambient Intelligence and Humanized Computing, vol. 10, pp. 321-332, 2019. https://doi.org/10.1007/s12652-018-0703-9.

[29] J. Andelmin and E. Bartolini, "A multi-start local search heuristic for the Green Vehicle Routing Problem based on a multigraph reformulation," Computers \& 
$\begin{array}{llllll}\text { Operations } & \text { Research, } & \text { vol. } & 109, & \text { pp. } & 43-63,\end{array}$ https://doi.org/10.1016/j.cor.2019.04.018.

[30] G. Macrina, G. Laporte, F. Guerriero, and L. Di Puglia Pugliese, "An energyefficient green-vehicle routing problem with mixed vehicle fleet, partial battery recharging and time windows," European Journal of Operational Research, vol. 276, pp. 971-982, 2019. https://doi.org/10.1016/j.ejor.2019.01.067.

[31] L. Wang and J. Lu, "A memetic algorithm with competition for the capacitated green vehicle routing problem," IEEE/CAA Journal of Automatica Sinica, vol. 6, pp. 516-526, 2019. https://doi.org/10.1109/JAS.2019.1911405.

[32] J. Zhang, Y. Zhao, W. Xue, and J. Li, "Vehicle routing problem with fuel consumption and carbon emission," International Journal of Production Economics, vol. 170, pp. 234-242, 2015. https://doi.org/10.1016/j.ijpe.2015.09.031.

[33] I. C. Trelea, "The particle swarm optimization algorithm: convergence analysis and parameter selection," Information Processing Letters, vol. 85, pp. 317-325, 2003. https://doi.org/10.1016/S0020-0190(02)00447-7.

[34] R. C. Eberhart and S. Yuhui, "Tracking and optimizing dynamic systems with particle swarms," in Proceedings of the 2001 Congress on Evolutionary Computation (IEEE Cat. No.01TH8546), 2001, pp. 94-100 vol. 1. https://doi.org/10.1109/CEC.2001.934376.

[35] A. Ratnaweera, S. K. Halgamuge, and H. C. Watson, "Self-organizing hierarchical particle swarm optimizer with time-varying acceleration coefficients," IEEE Transactions on Evolutionary Computation, vol. 8, pp. 240-255, 2004. https://doi.org/10.1109/TEVC.2004.826071.

[36] T. J. Gaskell, "Bases for Vehicle Fleet Scheduling," Journal of the Operational Research Society, vol. 18, pp. 281-295, 1967. https://doi.org/10.1057/jors.1967.44.

[37] G. B. Dantzig and J. H. Ramser, "The truck dispatching problem," Management science, vol. 6, pp. 80-91, 1959. https://doi.org/10.1287/mnsc.6.1.80. 